\section{LA-UR- $94-4118$}

Title:

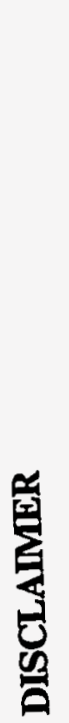

Submitted to:

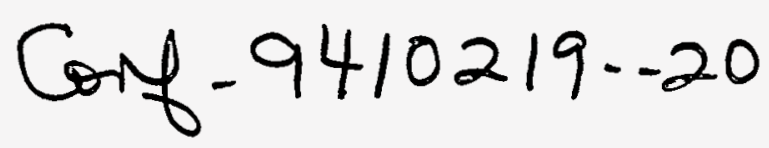

Optical and x-ray Imaging of Electron Beams Using Synchrotron Emission
M. D. Wilke

\section{RECEIVED DEC 051994 OSTI}
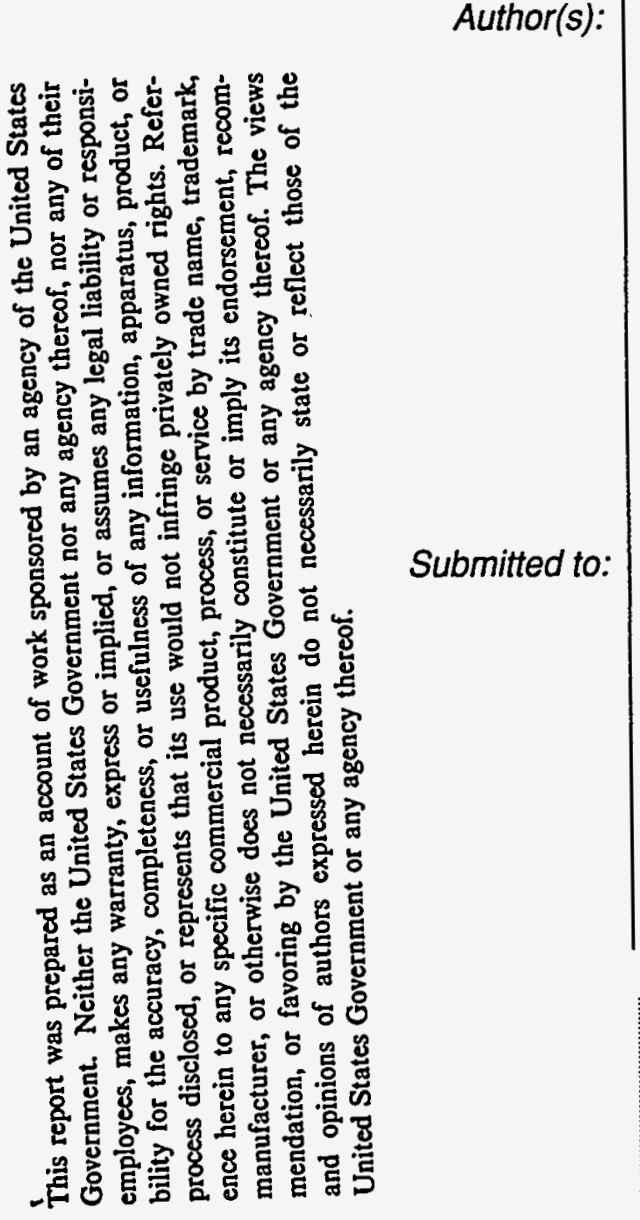

Proceedings of Beam Instrumentation Workshop

October 2-6, 1994

Vancouver, BC

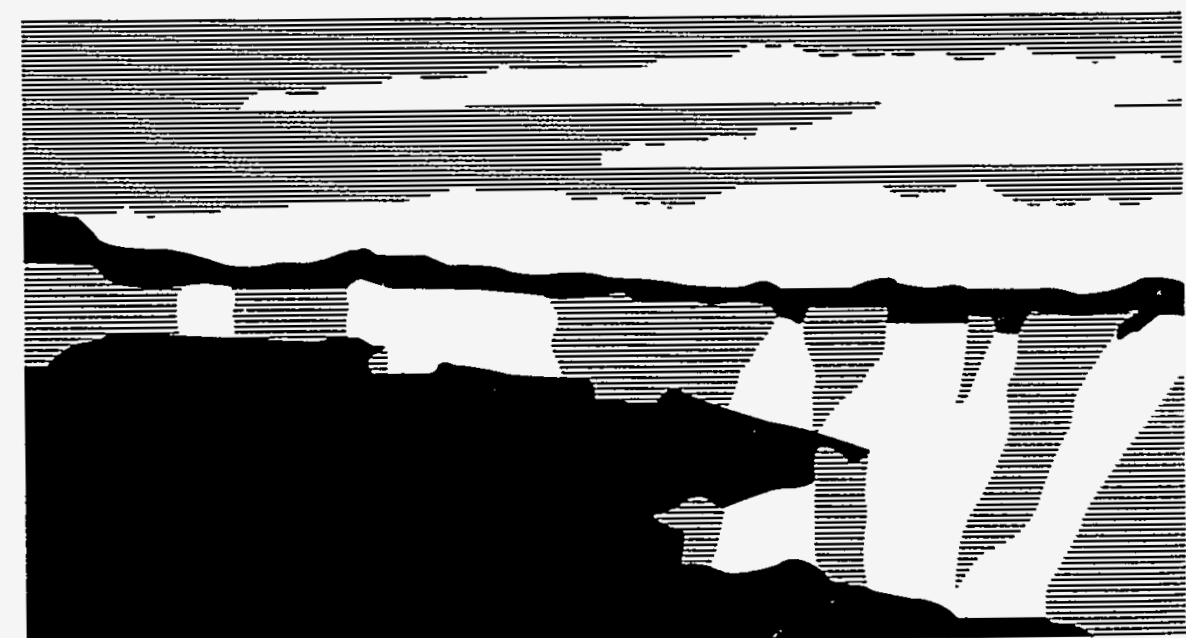




\section{DISCLAIMER}

Portions of this document may be illegible in electronic image products. Images are produced from the best available original document. 


\title{
Optical and X-ray Imaging of Electron Beams Using Synchrotron Emission *
}

\author{
Mark Wilke \\ Los Alamos National Laboratory, Los Alamos, NM 87545
}

\begin{abstract}
In the case of very low emittance electron and positron storage ring beams, it is impossible to make intrusive measurements of beam properties without increasing the emittance and possibly disrupting the beam. In cases where electron or positron beams have high average power densities (such as free electron laser linacs), intrusive probes such as wires and optical transition radiation screens or Cherenkov emitting screens can be easily damaged or destroyed. The optical and $\mathrm{x}$-ray emissions from the bends in the storage rings and often from linac bending magnets can be used to image the beam profile to obtain emittance information about the beam. The techniques, advantages and limitations of using both optical and x-ray synchrotron emission to measure beam properties are discussed and the possibility of single bunch imaging is considered. The properties of suitable imagers and converters such as phosphors are described. Examples of previous, existing and planned applications are given where available, including a pinhole imaging system currently being designed for the Advanced Photon Source at Argonne National Laboratory.
\end{abstract}

\section{INTRODUCTION}

The imaging of synchrotron radiation provides a useful alternative to invasive techniques for determining electron and positron beam profiles. In the case of storage ring beams, the use of wires, or Cherenkov or optical transition screens will disrupt the beam. High-intensity electron beams in high duty factor electron linacs, such as those used for free electron laser applications, will quickly damage the screens or wires. The synchrotron imaging technique also provides a direct measure of the electron beam profile, as opposed to methods such as beam collision techniques (which requires a second beam or a probe beam) or estimates of beam diameters from beam lifetime calculations.

Synchrotron light has been considered for use as a beam diagnostic since the first observation in 1947(1). As higher energy light sources have been constructed, diagnostic methods have been developed using visible through $\mathrm{x}$-ray radiation. Techniques tend either to measure the distribution and direction of the angular distribution of the synchrotron radiation to infer a beam emittance or to directly

* Work performed under the auspices of the Department of Energy 
image the beam bunch using an appropriate optical technique for the type of emission being imaged(2-7).

This paper discusses the imaging technique in both the visible-ultraviolet and the $\mathrm{x}$-ray portions of the spectrum for measuring beam profiles. Included are descriptions of the methods, advantages, and limitations of the technique.

\section{OPTICAL SYNCHROTRON RADIATION IMAGING}

\section{Analytical Optical Resolution Estimates}

The following derivations of resolution use the definitions of Jackson for critical energy $E_{c}$

$$
E_{C}=\frac{h \omega_{C}}{2 \pi}=\frac{3 h \gamma^{3}}{2 \pi}\left(\frac{c}{\rho}\right)
$$

or, equivalently, the critical wavelength $\lambda_{c}$ given by

$$
\lambda_{c}=\frac{2 \pi \rho}{3 \gamma^{3}}
$$

where $\omega_{c}$ is the critical wavelength, $\gamma=1 /\left(1-(v / c)^{2}\right)^{1 / 2}, \rho$ is the radius of the bend defined in Fig. 1, $c$ is the speed of light, and $h$ is Planck's constant(8). It should be noted that Jackson's value of $E_{c}$ is two times the value used by many other authors.

Figure 1 is a schematic of an optical imaging system. An optical element of aperture diameter $D$ images the synchrotron radiation emitted by particles turning the bend of radius $\rho$ at approximately a distance $l_{s}$ from the bend through an angle of $\theta \sim D / l_{s}$. Particles emit radiation of a given wavelength $\lambda$ or equivalently energy $E$ into a cone of angle $\theta_{c}$ given by

$$
\begin{array}{cc}
\theta_{c} \cong \frac{1}{\gamma}\left(\frac{E_{c}}{E}\right)^{1 / 3}=\frac{1}{\gamma}\left(\frac{\lambda}{\lambda_{c}}\right)^{1 / 3} & E_{c} \gg E, \\
\theta_{c} \cong \frac{1}{\gamma} & E_{c} \cong E, \\
\theta_{c} \cong \frac{1}{\gamma}\left(\frac{E_{c}}{3 E}\right)^{1 / 2}=\frac{1}{\gamma}\left(\frac{\lambda}{3 \lambda_{c}}\right)^{1 / 2} & E_{c}<E .
\end{array}
$$


As shown in Fig. 1, for large $D$, the particle appears to have a horizontal width of $\Delta \rho \cong \rho \theta^{2} / 8$ as it turns the bend. The particle is observed for a time $t_{D}=\rho \theta / c$. When

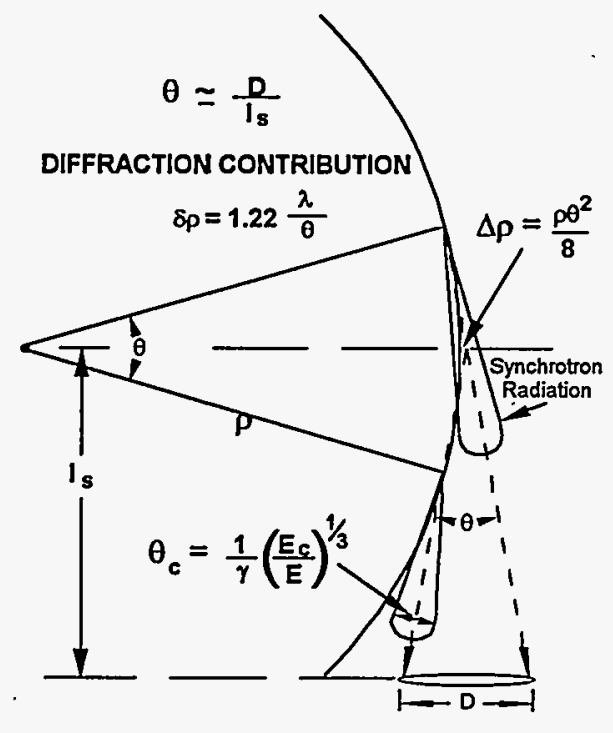

Figure 1. Schematic of optical system for imaging synchrotron radiation.

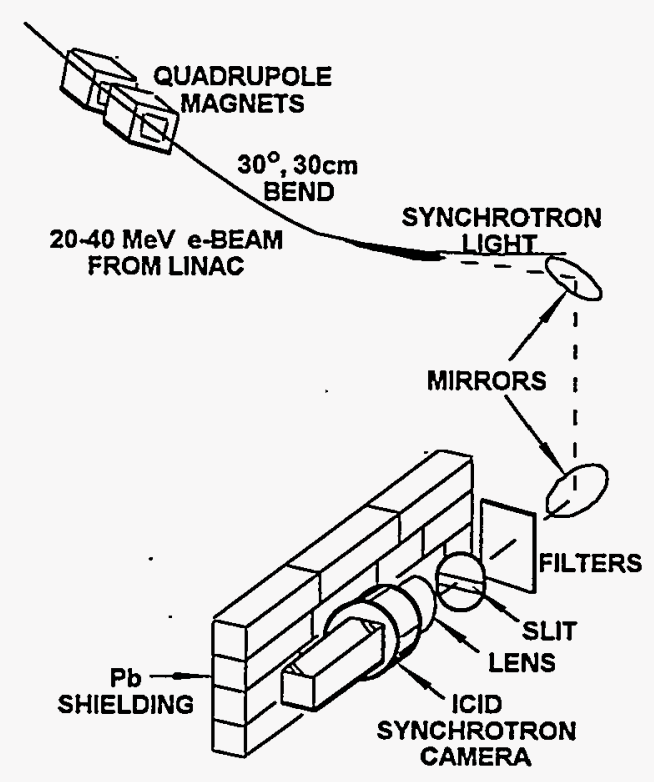

Figure 2. Schematic of the APEX linac visible synchrotron emission imaging system.

$D$ is small and the wavelength of observation $\lambda$ is long, diffraction becomes important and the particle appears to have an effective width $\delta \rho=1.22 \lambda / \theta$. The resolution of the imaging system resulting from the combined effects of the particle motion and diffraction is then given approximately by

$$
R=\sqrt{(\Delta \rho)^{2}+(\delta \rho)^{2}}=\sqrt{\left(\frac{\rho \theta^{2}}{8}\right)^{2}+\left(1.22 \frac{\lambda}{\theta}\right)^{2}} .
$$

The optimum value of $\theta$ defined as $\theta_{o}$, and therefore the optimum value of $D$ given by $D_{o}=l_{s} \theta_{o}$, is found by minimizing $R$ with respect to $\theta$, and is given by

$$
\theta_{o}=1.9\left(\frac{\lambda}{\rho}\right)^{1 / 3} .
$$

Therefore, the optimized resolution, $R_{o}$, is given by 


$$
R_{o}=0.78\left(\rho \lambda^{2}\right)^{1 / 3}=0.78 \rho^{1 / 3}\left(\frac{h c}{E}\right)^{2 / 3}
$$

In order to compare $\theta_{o}$ with $\theta_{c}$, we can note that the optics system will image at wavelengths in the visible so that $\lambda \gg \lambda_{c}\left(E \ll E_{c}\right)$. Substituting Eq. 2 for $\lambda_{c}$ in Eq. 3a gives

$$
\theta_{c}=\left(\frac{3}{2 \pi} \frac{\lambda}{\rho}\right)^{1 / 3}=0.78\left(\frac{\lambda}{\rho}\right)^{1 / 3},
$$

which by Eq. 4 gives the resolution $R_{c}$ of an optical system when the aperture is $D=l_{s} \theta_{c}$

$$
R_{c}=1.56\left(\rho \lambda^{2}\right)^{1 / 3}=1.56 \rho^{1 / 3}\left(\frac{h c}{E}\right)^{2 / 3} .
$$

The resolution therefore appears to be worse than $R_{0}$ by a factor of two. The instantaneous cone implies a resolution given by Eq. 8. However, analogous to the enhancement of resolution in synthetic aperture radar, the horizontal resolution of an image produced by the synchrotron radiation beam scanning the whole aperture is given by Eq. 6 and not Eq. 8. The approximate vertical resolution is given by Eq. 8 .

Above $E_{c}$, the emitted synchrotron power per unit frequency falls rapidly. We may define an approximate limit to the achievable resolution of an optics system, which is determined by the usable signal level. When $E \cong E_{c}$, so that from Eq. 3b $\theta \sim 1 / \gamma$, the major contribution to $R$ is from the diffraction term $\delta \rho$. If one substitutes $\Delta \rho$ and $\delta \rho$ in terms of $\theta$ into Eq. 4 and sets $\theta=1 / \gamma$ and $\lambda=\lambda_{c}$ as given by Eq. 2 , then the defined limiting resolution $R_{l}$ of an optics system that images a synchrotron beam at the critical energy is given by

$$
R_{l}=2.6 \frac{\rho}{\gamma^{2}} .
$$

There is also a technological limit to the achievable resolution, because as $\lambda$ becomes shorter, it becomes more difficult to produce diffraction-limited optics.

Table 1 lists values of several of the parameters defined above, including the resolution of an optical system imaging the beam at $\lambda=500 \mathrm{~nm}$ for two cases: a $30 \mathrm{~cm}$ bend in a linac where the beam energy $E_{b}=40 \mathrm{MeV}$ and a $3900 \mathrm{~cm}$ bend in a high energy synchrotron where $E_{b}=7000 \mathrm{MeV}$.

In the first case, $\lambda_{c}>\lambda=500 \mathrm{~nm}$, and Eq. $3 \mathrm{c}$ was used to calculate $\theta_{c}$. This value of $\theta_{c}$ was then used to derive an equation for $R_{c}$ in a way similar to that used 
in deriving Eq. 8. In the second case, where $\lambda \gg \lambda_{c}$, Eq. 3a was used to calculate $\theta_{c}$ and Eq. 8. was used to calculate $R_{c}$. From Table 1 it can be seen that for the first case; $\theta_{o}$ is 4.6 times larger than $\theta_{c}$. One can therefore expect an improvement in the horizontal resolution of the beam by using a lens of aperture $D \approx l_{s} \theta_{o}$, but the vertical beam resolution will be diffraction limited by $\theta_{c}$ to $R_{c}=133 \mu \mathrm{m}$.

Table 1. Calculated parameters of a system imaging an electron beam at $\lambda=500 \mathrm{~nm}$.

\begin{tabular}{|c|c|c|c|c|c|c|c|c|}
\hline$\gamma$ & $\begin{array}{c}\lambda_{\mathrm{c}} \\
(\mathrm{nm})\end{array}$ & $\begin{array}{c}E_{c} \\
(\mathrm{eV})\end{array}$ & $\begin{array}{c}\theta_{0} \\
(\mathrm{rad})\end{array}$ & $\begin{array}{c}\theta_{\mathrm{c}} \\
(\mathrm{rad})\end{array}$ & $\begin{array}{c}t_{D} \\
\text { (ps) }\end{array}$ & $\begin{array}{c}R_{\mathrm{o}} \\
(\mu \mathrm{m})\end{array}$ & $\begin{array}{c}R_{\mathrm{c}} \\
(\mu \mathrm{m})\end{array}$ & $\begin{array}{c}R_{l} \approx 2.6 \rho / \gamma^{2} \\
(\mu \mathrm{m})\end{array}$ \\
\hline & & & \multicolumn{2}{|c|}{$E_{b}=40 \mathrm{MeV}$} & \multicolumn{2}{|c|}{$\rho=30 \mathrm{~cm}$} & & \\
\hline 79 & 1261 & 0.984 & 0.023 & 0.005 & 23 & 30 & 133 & 95 \\
\hline \multicolumn{5}{|c|}{$E_{b}=7000 \mathrm{MeV}$} & $\rho=3 \mathrm{~s}$ & $00 \mathrm{~cm}$ & & \\
\hline 13700 & 0.032 & 39058 & 0.004 & 0.002 & 578 & 151 & 302 & 0.42 \\
\hline
\end{tabular}

As defined here, $\theta_{c}$ is conservative. The angular distribution of the synchrotron radiation has a large portion of the total power in the wings. Also in the first case (where $\lambda_{c}>\lambda$ ), the value of $\theta_{c}$ more nearly represents the value from the peak to the $1 / e$ value of the distribution, and not the full-width at half-maximum (FWHM) value. The value of $\theta_{o}$ is only twice as great as that of $\theta_{c}$ in the second case.

There are several relevant time scales. Imaging systems such as cameras typically have interrogation times on the order of $10^{-2}$ to 10 seconds, and are therefore time integrating devices. Linac macro-pulse lengths and synchrotron orbit times are on the order of microseconds while the micro-pulse separations may be $10^{-8}$ to $10^{-7}$ seconds. Micro-pulse lengths may be in the picosecond regime, but the use of synchrotron light to observe micro pulse is limited by the time the synchrotron light illuminates the imaging system $\left(t_{D}\right)$, which is typically greater than micro-pulse lengths, as seen in Table 1.

Finally, we note in the second case that the resolution of $151 \mu \mathrm{m}$ at $500 \mathrm{~nm}$ can be seen to be considerably worse than the $0.42 \mu \mathrm{m}$ that would be achievable if diffraction-limited optics were available at the critical energy of $30 \mathrm{keV}$.

\section{Optical Imaging of a Linac Beam}

Figure 2 shows a system used to image an electron beam in a bend of the APLE Protoype Experiment (APEX) via optical synchrotron emission. APEX was a free electron laser driven by a linac which could be run at 20 to $40 \operatorname{Mev}(9)$. The beam format typically consisted of 10 to $15 \mathrm{psec}, 1$ to $5 \mathrm{nC}$ micro pulses from a photo injector spaced that were typically $47 \mathrm{nsec}$ apart. The macro pulse could be varied from a single micro pulse to a pulse $100 \mu$ s long containing a maximum of $10 \mu \mathrm{C}$. 
The beam was directed to the laser wiggler by a 60 deg achromatic, isochronous bend composed of two $30 \mathrm{deg}$ bending magnets. The beam was matched to the bend by focusing the beam to a horizontal waist halfway through each of the $30 \mathrm{~cm}$ radius, 30 deg bending magnets.

Glass windows were located at each bend in order to determine the beam profile and trajectory via the synchrotron emission. A periscope configuration of mirrors relayed the light to the shielded intensified CID camera (ICID). The sensitivity of the S20-response ICID at full intensifier gain was $1.2 \times 10^{9} \mathrm{~V} / \mathrm{J} / \mathrm{cm}^{2}$ at $500 \mathrm{~nm}$ with a camera noise level of $10 \mathrm{mV}$. The view was limited by the lens aperture to $48 \mathrm{mrad}$ in the vertical direction and to $5 \mathrm{mrad}$ in the horizontal direction by the $0.5 \mathrm{~cm}$ slit placed in front of the lens. Therefore, the horizontal resolution was diffraction limited to $133 \mu \mathrm{m}$. The slit was originally selected to match $\theta_{c}$. As Table 1 indicates, matching $\theta_{o}$ would have given $30 \mu \mathrm{m}$ resolution. The camera recorded images on video tape, and the images were later digitized for analysis.

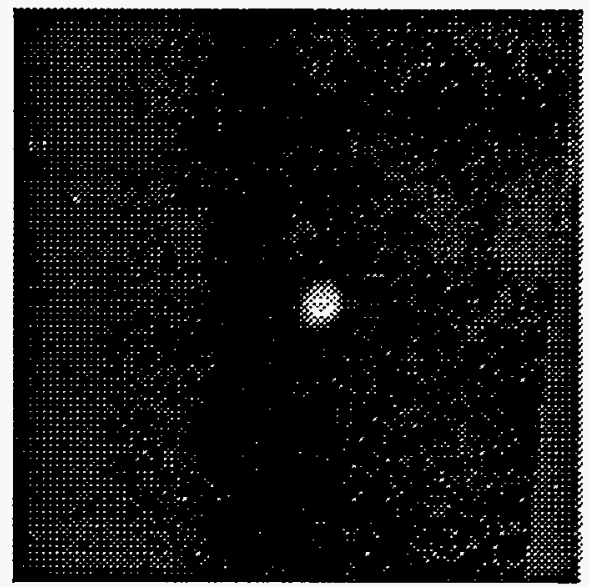

Figure 3a

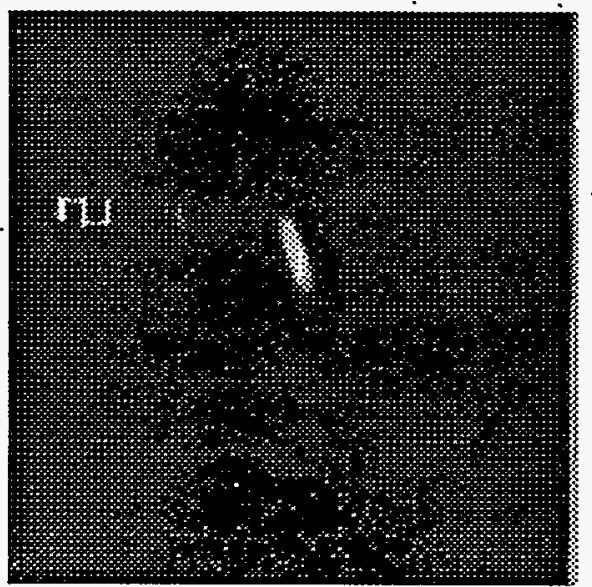

Figure $3 b$

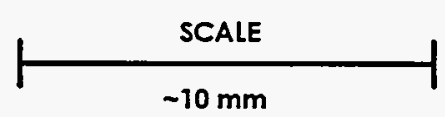

Figure 3. Two synchrotron emission images of the Linac beam. a) Beam focused in both directions. b) Beam with an optimal horizontal focus.

Two images from the camera are shown in Fig. 3. In Fig. 3a, the $38 \mathrm{MeV}$ beam was focused in both the horizontal and vertical directions. The FWHM was $765 \mu \mathrm{m}$ in both directions. In Fig. 3b; the beam was optimally focused in the horizontal direction, which resulted in a horizontal FWHM of $382 \mu \mathrm{m}$ and generally produced 
better lasing. Note that the beam is slightly tipped. The major axis of the elliptical beam in Fig. $3 \mathrm{~b}$ was $1.6 \mathrm{~mm}$.

To determine how low a beam energy this diagnostic would be useful for, images were taken as $E_{b}$ was varied. Figure 4 is a plot of the camera peak signal normalized to the total macro-pulse charge. For this camera, the noise floor was about $4 \times 10^{3} \mathrm{~V} / \mathrm{C}$. The signal was also calculated from the synchrotron energy radiated per unit frequency per solid angle, knowing the geometry and response of the imaging system. The calculated response is given as the dashed curve in Fig. 4. The signal decreases abruptly at low $E_{b}$, showing that imaging synchrotron radiation in the visible range is no longer practical for these beam conditions for beam energies below about $23 \mathrm{MeV}$.

\section{SYNCHROTRON SIGNAL vs BEAM ENERGY FOR $30 \mathrm{~cm}$ BEND}

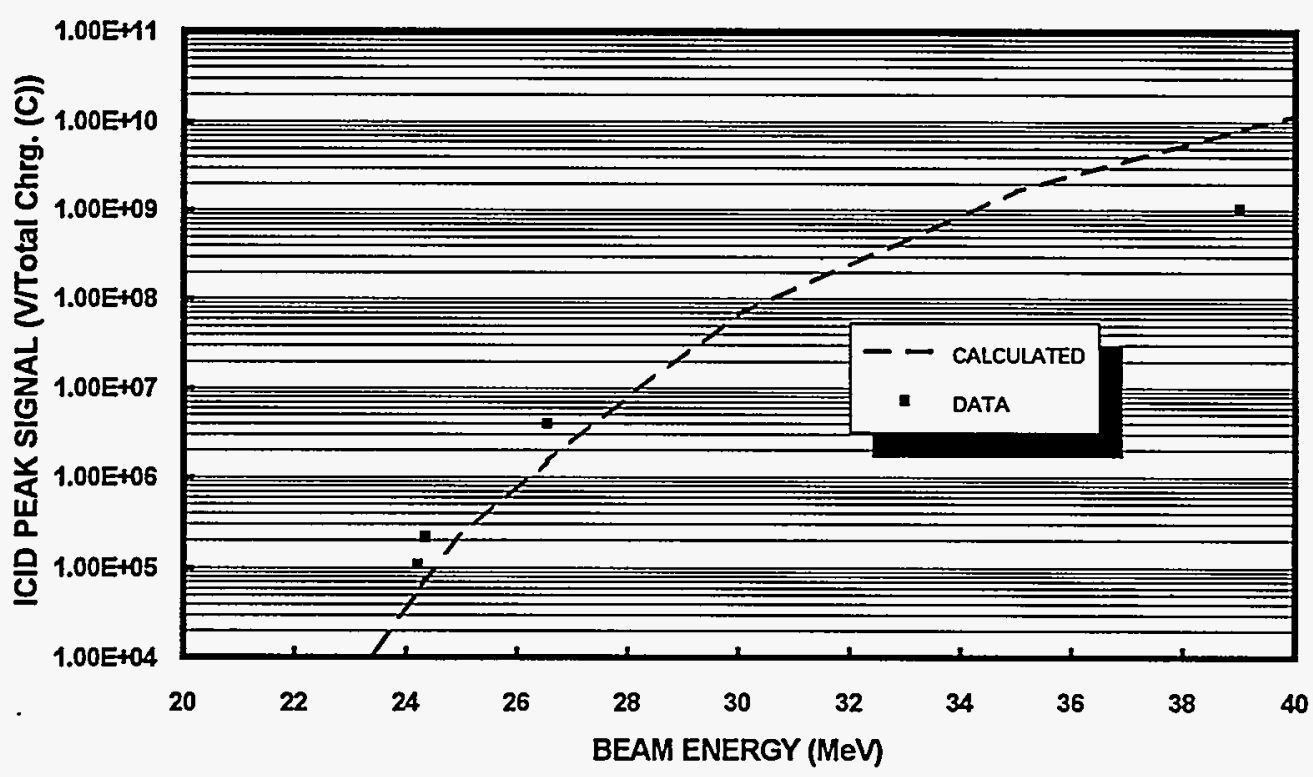

Figure 4. Plot of charge-normalized synchrotron image signal vs. beam energy.

\section{X-RAY SYNCHROTRON RADIATION IMAGING}

Although it is difficult to construct diffraction-limited optical elements that are useful at high x-ray energies, it is possible to build pinholes for imaging. In the case of pinhole imaging, there is a tradeoff between geometrical blurring due to the finite size of the pinhole and loss of resolution due to diffraction. By imaging at high 
energies, diffraction is minimized. This permits the use of pinholes small enough to overcome geometrical blurring. Therefore, in order to have adequate signal, the use of pinhole imaging of high-brightness beams is limited to high-energy, high-current synchrotrons.

\section{Analytical X-Ray Pinhole Resolution Estimates}

Figure 5 illustrates the geometrical blurring of the image due to the finite size of the pinhole. Two points within the beam, separated by $\Delta R$, generate two circular images of radii $r_{i}$ at the image plane when projected through a pinhole of diameter $D$. The two images are defined as resolved when separated by a distance $\Delta R_{i} \geq C r_{i}$ where $C$ is a constant between 1 and 2 defined by some resolution criterion. Here we define $\Delta R_{i}$ as the distance between the two circles when the secant of the intercept region of the two circles is equal to $r_{i}$, as shown in Fig. 5. Therefore $C=\sqrt{3}$. It can be seen from Fig. 5 that the various parameters can be related by

$$
\Delta R=\frac{l_{o} \Delta R_{i}}{l_{i}}=\frac{C r_{i}}{M}=\frac{1+M}{M} C D,
$$

where $M=l_{i} l_{s}$ is the magnification of the pinhole.
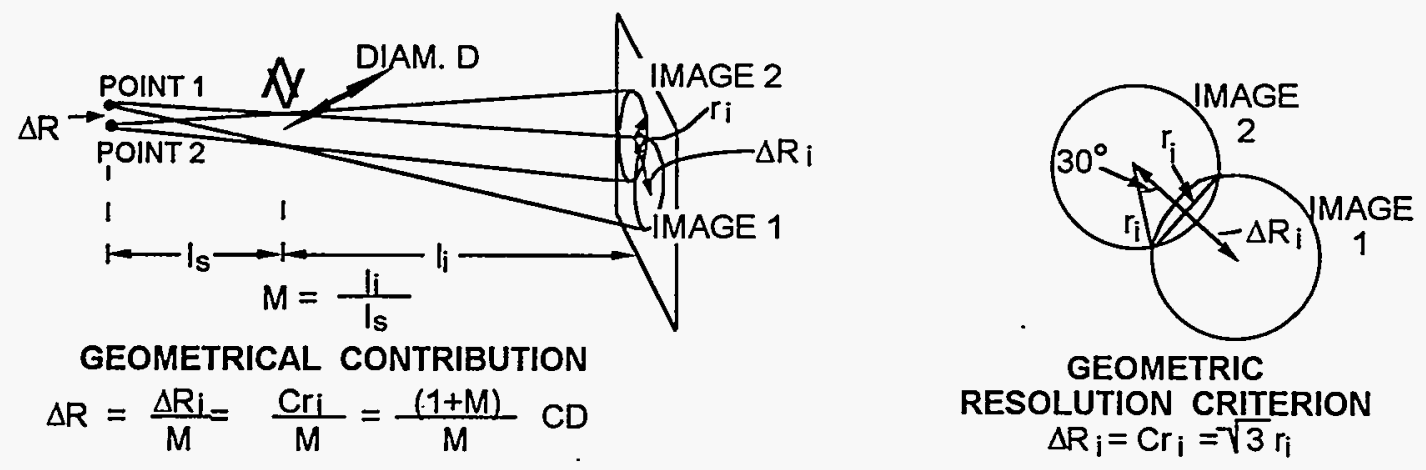

Figure 5. Schematic defining various pinhole imaging parameters.

The diffraction contribution to the blurring of the image is again $\delta \rho=1.22 \lambda / \theta=1.22 \lambda l_{s} / D=1.22 \mathrm{hc} / E D$. There is also a contribution equivalent to $\Delta \rho$ from apparent motion of the particle as it rounds the bend while the synchrotron radiation sweeps over the pinhole. For large $\gamma, \Delta \rho$ is fractions of a micron and will be ignored. Therefore, the resolution $R$ that is possible using a pinhole of aperture $D$ is given by 


$$
R=\sqrt{\left(\frac{1+M}{M} C D\right)^{2}+\left(1.22 \frac{h c}{E} \frac{l_{s}}{D}\right)^{2}} .
$$

The optimized resolution, $R_{0}$, for the optimum pinhole diameter $D_{o}$, at a given photon energy $E$, is found by solving for $D_{o}$ after differentiating Eq. 11 with respect to $D_{o}$ and setting the result equal to zero. The optimum values are then

$$
\begin{gathered}
D_{o}=\sqrt{1.22 \frac{M l_{s}}{1+M} \frac{h c}{C E}} \text { and } \\
R_{o}=\sqrt{2} \frac{1+M}{M} C D_{o}=\sqrt{2.44 l_{s} C \frac{1+M}{M} \frac{h c}{E}} .
\end{gathered}
$$

For reasonable values of $M$ in the range of 1 to 4 , there is little $M$ dependence. Both $D_{o}$ and $R_{o}$ vary as $E^{-1 / 2}$, so that imaging at higher energies yields a corresponding improvement in resolution. Table 2 lists values of $D_{o}$ and $R_{o}$ for different values of $E$ and $M$ for $l_{s}=1400 \mathrm{~cm}$. Comparison with Table 1 shows that a pinhole imaging system that images a high-energy synchrotron will have improved resolution compared to a system imaging at $500 \mathrm{~nm}$.

Table 2. Values of $D_{o}$ and $R_{o}$ for different values of $E$ and $M$ for $l_{s}=1400 \mathrm{~cm}$.

\begin{tabular}{l|cc|cc}
\hline \hline & \multicolumn{2}{|c|}{$E=39058 \mathrm{eV}$} & \multicolumn{2}{c}{$E=80000 \mathrm{eV}$} \\
& $M=1$ & $M=4$ & $M=1$ & $M=4$ \\
\hline$D_{o}$ & 12.5 & 15.8 & 8.7 & 11.0 \\
$R_{o}$ & 61.2 & 48.4 & 42.8 & 33.8 \\
\hline \hline
\end{tabular}

\section{Experimental considerations}

Figure 6 is a schematic of a synchrotron pinhole imaging system showing the various elements involved.

Pinholes with the optimum pinhole diameters given in Table 2 are technologically challenging to fabricate. Pinhole imaging, at the higher $\mathrm{x}$-rays energies that are required to achieve better than $100 \mu \mathrm{m}$ resolution, requires high- $Z$ pinholes of several millimeters thickness. Crossed slits may also be used in place of a pinhole. A crossed-slit beam diagnostic is currently under test at the Brookhaven National Light Source.(5)

High aspect Au pinholes $50 \mu \mathrm{m}$ in diameter and several inches long for neutron pinhole imaging have been produced at Los Alamos National Laboratory by plating $\mathrm{Au}$ on $\mathrm{Cu}$ wire and acid etching away the wire mandrel(10). It is difficult to obtain 
round wires with diameters on the order of $10 \mu \mathrm{m}$ for. use as mandrels, but some manufactures will supply short lengths suitable for pinhole fabrication. Plasma etching techniques can be used for reducing the diameter of thicker wires. Carbon wires with $8 \mu \mathrm{m}$ diameters are commercially available but tend not to be round. Carbon wires require a sputtered metal coating before Au can be chemically plated. A $2.8 \mathrm{~mm}$ thick, $10 \mu \mathrm{m}$ diameter $\mathrm{Au}$ pinhole is currently being designed for the Advanced Photon Source (APS). The APS pinhole will be fabricated using the mandrel technique by plating the $\mathrm{Au}$ on a $10 \mu \mathrm{m} \mathrm{Cu}$ wire.
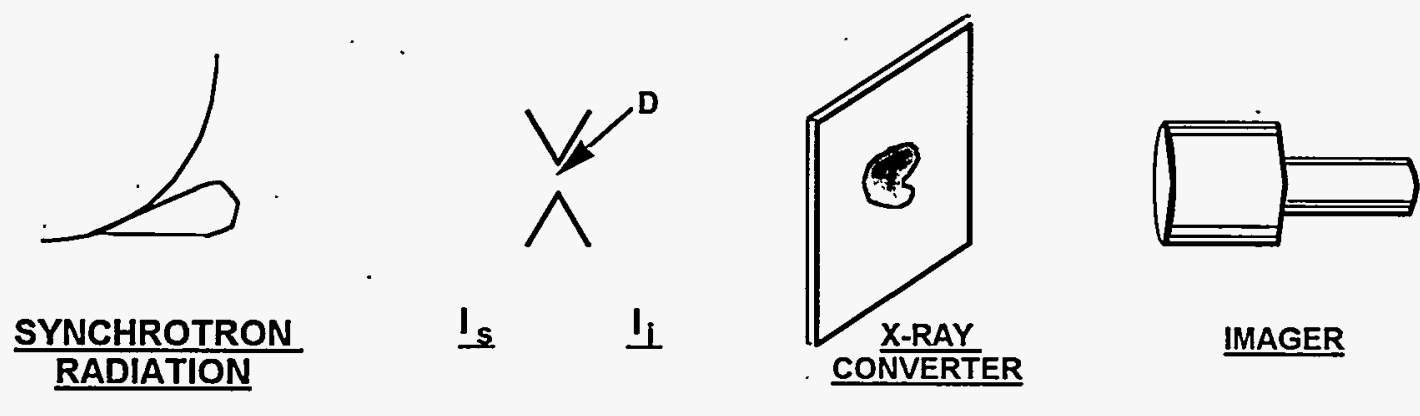

IMAGER

Figure 6. A schematic of a synchrotron pinhole imaging system.

Characterization of a small diameter pinhole is also difficult. Continuous x-ray tube sources exist that produce source sizes of several microns. Energies are on the order of $150 \mathrm{keV}$, so diffraction is minimized. However, neither this energy nor source size is sufficient to eliminate consideration of the calibration source dimensions and beam diffraction from the pinhole characterization.

As with any object in a high-energy synchrotron $x$-ray beam, pinhole heating is a concern. Electroplated $\mathrm{Au}$ alloys are typically more sensitive to heat than other $\mathrm{Au}$ alloys. The $\mathrm{Au}$ begins to distort at temperatures as low as 300 to $400 \mathrm{deg} \mathrm{C}$ because of trace impurities. Standard procedures that pre-collimate and pre-filter the x-ray beam and cool the pinhole can all be used to mitigate heating. As an example, finite-element heat transport modeling of pinhole designs was done for the APS pinhole. At $1400 \mathrm{~cm}$, the peak design flux for the APS pinhole is $20 \mathrm{~W} / \mathrm{mm}^{2}$. The X-ray beam was filtered with $5 \mathrm{~mm}$ of Be and $2 \mathrm{~mm}$ of $\mathrm{Al}$ so that the spectrum peaked at $30 \mathrm{keV}$, and the $\mathrm{x}$-rays were collimated through a $0.5 \mathrm{~cm}$ diameter aperture. Calculations gave temperatures below $150 \mathrm{deg} \mathrm{C}$ in both the filter stack and pinhole assembly. when the assembly was cooled to $23 \mathrm{deg} C$ at a radius of 2 $\mathrm{cm}$. The X-ray beam could be collimated further to reduce the total incident power to less than 1 watt at the pinhole, making it possible to avoid having to liquid cool the pinhole assembly. Extreme collimation of the x-rays, however, makes alignment of the system difficult. 
Several possibilities exist for visualizing the pinhole image. Figure 6 shows an image converter such as a phosphor recorded with an electrooptic imager. In all cases, a major concern is the image size. Reasonable magnifications still produce beam images with dimensions of less than $1 \mathrm{~mm}$. Therefore, the inherent resolution of any converter must be considered. A second major concern is the effect of the radiation on the converter. The pinhole geometry and spectral filtering reduces the dose, but the integrated dose can be substantial if the diagnostic is used for long periods. Use of a shutter can help.

Film provides the simplest method of visualization. Due to development time, film is not a real-time method suitable for monitoring beam conditions. A somewhat faster method is to scan the pinhole image plane with a detector. If a slit is used, tomographic views of the beam profile may be obtained. By rotating the slit to obtain several beam profiles, a 2-D image can be generated in almost real time.

Direct electrooptic imaging of the beam is difficult because of the duty factor and energies of the beam. Direct $x$-ray imaging with charge-coupled-device (CCD) cameras has proven useful at low $x$-ray energies, and low-cost cameras have now been evaluated(11). Front side CCD illumination is possible above fractions of a $\mathrm{keV}$. Above about $10 \mathrm{keV}$ the $\mathrm{CCD}$ becomes increasingly transparent to the $\mathrm{x}$-rays and quantum efficiency decreases rapidly. Further difficulties arise from finite CCD well sizes and from radiation damage over long periods of use. A CCD charge well will accommodate $\gtrsim 10^{5}$ electrons. One electron-hole pair is generated per $3.65 \mathrm{eV}$. An $80 \mathrm{keV} x$-ray can generate $>2 \times 10^{4}$ electrons per well and several hits will saturate the well. Therefore, dynamic range is limited. When integrated doses approach several krad, permanent damage becomes apparent even for CCDs using multipin phasing. The CCD noise level begins to increase, further limiting dynamic range(12).

Microchannel plate image intensifiers (MCPIIs) can also be used to detect the $\mathrm{X}$-rays. Generally, the input side of the MCPII is coated with an $\sim 1 \mu \mathrm{m}$ thick, high$Z$ photocathode such as Au. The MCPII can then be turned off and on by controlling the voltage across the MCP. Above about $30 \mathrm{keV}$, the direct excitation of the phosphor by $\mathrm{x}$-rays penetrating the $\mathrm{Au}$ and MCP gives a signal comparable to that from the Au photocathode.

Phosphors imaged with an electrooptic device provide a simple method for observing the $x$-ray image, but for very small images, phosphor grain sizes typically a few microns may complicate resolution. Organic scintillators also may be used, but in general the organics become nonlinear and sustain radiation damage at lower dose levels than inorganics. Organic scintillators also have a lower $\mathrm{Z}$ and therefore, although they are typically fast and more efficient at converting radiation to light, they are more transparent to high-energy $x$-rays. High- $Z$ loaded liquid scintillators have shown some promise for use as fast, bright, $x$-ray-to-light converters(13). Because of self-absorption and internal scatter, phosphor screens must be thin. Therefore, phosphors containing high-Z elements such as $\mathrm{Gd}, \mathrm{Y}$, and $\mathrm{Eu}$, and scintillators such as CsI are often used. These phosphors generally show long decay 
times of tens to hundreds of microseconds and often have long lag components on the.order of milliseconds $(14,15)$. Some of the inorganics have also been evaluated at high $\mathrm{x}$-ray doses $(16)$. Ruby $\left(\mathrm{Al}_{2} \mathrm{O}_{3}: \mathrm{Cr}^{3+}\right)$ screens used for direct accelerator beam monitoring are also useful for $\mathrm{x}$-ray applications.

Table 3. Properties of some common phosphors and two fast phosphors.

\begin{tabular}{|c|c|c|c|c|}
\hline $\begin{array}{l}\text { PHOSPHOR } \\
\text { TYPE }\end{array}$ & $\begin{array}{c}\text { PEAK } \\
\text { WAVELENGTH }\end{array}$ & $\begin{array}{c}\text { DECAY TIME } \\
\text { to } 10 \% \\
(\mu \mathrm{sec}) \\
\end{array}$ & $\begin{array}{c}\text { POWER } \\
\text { EFFICIENCY } \\
(\mathrm{W} / \mathrm{W}) \\
\end{array}$ & COLOR \\
\hline $\mathrm{P}-11$ (BE) & 460 & $70-80$ & $\begin{array}{l}0.089 \\
\end{array}$ & Purple/Blue \\
\hline P-15 (GG) & $390 / 530$ & $2.6-2.8$ & 0.044 & Green \\
\hline P-16 (M) & 380 & 0.12 & 0.05 & UV \\
\hline $\mathrm{P}-20$ (KA) & 560 & $500^{\mathrm{a}}$ & 0.14 & Yellow/Green \\
\hline P-22 R & 680 & 1000 & 0.14 & Red \\
\hline P-RED & 680 & (See b) & $0.063^{\mathrm{c}}$ & Red \\
\hline $\mathrm{P}-24$ (GE) & 510 & 1.5 & 0.024 & Green \\
\hline P-31 (BH) & 520 & $30-35$ & $0.060^{\mathrm{c}}$ & Green \\
\hline $\mathrm{P}-36(\mathrm{KF})$ & 550 & 0.25 & (See b) & Yellow/Green \\
\hline P-46 (KG) & 540 & 0.16 & 0.010 & Yellow/Green \\
\hline $\mathrm{P}-47$ (BH) & 420 & 0.08 & 0.07 & Purple/Blue \\
\hline P-48 (KH) & $420 / 540$ & 0.12 & 0.06 & Yellow/Green \\
\hline WL-1201 & 395 & 0.0008 & 0.011 & Purple/Blue \\
\hline WL-1198 & 520 & 0.00013 & 0.001 & Green \\
\hline
\end{tabular}

NOTES: a - Varies with current density

b - Data not available

c - Data accuracy is questionable

Information regarding phosphors with $\mathrm{P}$-designations can be found in various handbooks(17). Most of the available information is pertinent to kilovolt electron beam excitation, because of its importance to cathode ray tubes. Even so, the information is difficult to correlate. Phosphors often emit at several wavelengths. The various bands of a given phosphor may have different time responses and brightness for different types and levels of excitation. Activator doping levels also influence behavior and may vary, depending on the manufacturer. Phosphors are almost always produced as powders. Methods for forming screens include combining the phosphor with organic binders, settling; plasma spraying, and in situ growth. The various methods cause wide fluctuations in a given phosphor's properties, especially for x-ray excitation. Publications typically give relative phosphor properties, especially conversion-to-light efficiencies, for a given type of excitation, screen thickness and geometry. Therefore, it is best to test a phosphor, particularly its efficiency, specifically for a given application. With these caveats, Table 3 is provided, listing some properties of the more common phosphors(18).

Table 3 also contains the properties of two subnanosecond phosphors that have been extensively evaluated for pulsed $x$-ray spectroscopy applications. The efficiencies listed for the P-designation phosphors are for electron excitation at 
optimal thickness and beam energy. The efficiencies for the WL-designation phosphors are absolute values of total emitted power into $4 \pi$ per absorbed x-ray power at $8 \mathrm{keV}$ measured at the Cornell synchrotron. Phosphor grain sizes, which are important indicator of a phosphor's resolution, are not listed. Grain sizes are typically on the order of one to several microns. Screen resolutions are also a function of the coating method used, because some techniques lead to clumps in the deposition.

Table 4. Properties of WL-1201 and WL-1198

\begin{tabular}{l|cc}
\hline \hline \multicolumn{1}{c|}{ COMPOSITION } & WL-1201 & WL-1198 \\
DENSITY & ZnO:Ga $(.003 \mathrm{~mole} \mathrm{Ga})$ & CdS:In $(.001 \mathrm{~mole}$ In) \\
EMISSION PEAK & $5.61 \mathrm{~g} / \mathrm{cm}^{3}$ & $4.82 \mathrm{~g} / \mathrm{cm}^{3}$ \\
FWHM & $390 \mathrm{~nm}$ & $520 \mathrm{~nm}$ \\
TIME RESPONSE: & $18 \mathrm{~nm}$ & $20 \mathrm{~nm}$ \\
$\quad$ FWHM & $730 \mathrm{ps}$ & \\
1/e DECAY & $800 \mathrm{ps}$ & $150 \mathrm{ps}$ \\
X-RAY CROSS SECTIONS: & & $130 \mathrm{ps}$ \\
$\quad$ 10 keV & $127 \mathrm{~cm}^{2} / \mathrm{g}$ & $102 \mathrm{~cm}^{2} / \mathrm{g}$ \\
$\quad 80 \mathrm{keV}$ & $0.6 \mathrm{~cm}^{2} / \mathrm{g}$ & $1.5 \mathrm{~cm}^{2} / \mathrm{g}$ \\
OPTICAL ABSORB. LNG. & $242 \mathrm{~cm}^{2} / \mathrm{g}$ & $150 \mathrm{~cm}^{2} / \mathrm{g}$ \\
GRAIN SIZE & $3.5 \mu \mathrm{m}$ & $3-25 \mu \mathrm{m}$ \\
LINEARITY AND & GOOD & $?$ \\
RAD HARDNESS & & \\
\hline \hline
\end{tabular}

Because there is little published literature on the properties of the WL-designation phosphors and because they are useful for pulse measurements, Table 4 is included which lists measured properties $(19,20)$. The properties of the WL phosphors can be modified by controlling the percentages of the activators. In particular, time response can be traded for efficiency.

Observation of the emission of the phosphor is a simpler problem than direct $\mathrm{x}$-ray observation. Where time resolution is not an issue, slow, bright phosphors can be imaged with standard television cameras, either vidicons or CCDs. Fast and/or less efficient phosphors require more sensitive cameras for time resolved imaging. High-quality, cooled CCD cameras are now capable of imaging at the tens of photons per pixel level. To resolve individual bunches requires some method of gating the imager, such as a gated MCPII. The MCPII also provides gain control, so that imaging at the several photons per pixel level is possible.

It is possible to estimate signal levels for an imager using the data from Table 3. Figure 7 is an illustration of a phosphor screen being excited by $\mathrm{x}$-rays incident with flux $P_{\not \supset}$. in W/ $/ \mathrm{cm}^{2}$ (or $\mathrm{J} / \mathrm{cm}^{2}$ for time-resolved single-bunch data). It is assumed that the phosphor grain size is small compared to the incremental excited volume. The

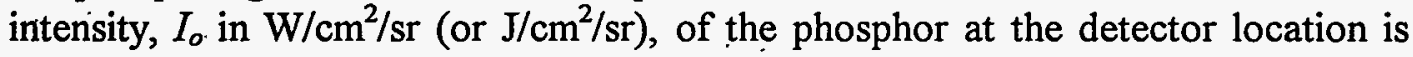
given by 


$$
\begin{aligned}
I_{o}= & \frac{P_{I x} C}{4 \pi} \sigma_{x} \cos \theta_{o} \exp \left(-\sigma_{o} \tau / \cos \theta_{o}\right) \\
& \left\{\frac{1}{\sigma_{o} \cos \varphi-\sigma_{x} \cos \theta_{o}}\left[\exp \left(\tau \frac{\sigma_{o} \cos \varphi-\sigma_{x} \cos \theta_{o}}{\cos \theta_{o} \cos \varphi}\right)-1\right]+\right. \\
& \left.\frac{-R}{\sigma_{o} \cos \varphi+\sigma_{x} \cos \theta_{o}}\left[\exp \left(-\tau \frac{\sigma_{o} \cos \varphi+\sigma_{x} \cos \theta_{o}}{\cos \theta_{o} \cos \varphi}\right)-1\right]\right\}
\end{aligned}
$$

Where $R$ is the reflectivity of an x-ray transparent substrate or reflector, $\theta_{0}$ and $\phi$ are defined in Fig. $7, \sigma_{0}$ and $\sigma_{\mathrm{x}}$ are the optical and $\mathrm{x}$-ray mass absorption coefficients in $\mathrm{cm}^{2} / \mathrm{g}$, and $T$ is the phosphor thickness in $\mathrm{g} / \mathrm{cm}^{2}$. $C$ is the efficiency in $\mathrm{W} / \mathrm{W}$ (or $\mathrm{J} / \mathrm{J}$ ). The values of $\sigma_{\mathrm{o}}$ and $C$ listed for the WL-phosphors in Table 4 were determined by fitting intensity data for samples with different values of $\tau$ excited by $\mathrm{x}$-rays from a monochrometer at CHESS. When $\theta_{0}=\phi=0$ degrees and the reflectivity is zero,

$$
\dot{I}_{o}=\frac{P_{\not \supset} C}{4 \pi} \frac{\sigma_{x}}{\sigma_{o}-\sigma_{x}}\left[\exp \left(-\sigma_{x} \tau\right)-\exp \left(-\sigma_{o} \tau\right)\right] .
$$

For completeness, when the detector is on the side of the phosphor illuminated by the $\mathrm{x}$-rays so that $90<\theta_{0} \leqq 180 \mathrm{deg}$, and the reflective substrate of reflectivity is on the opposite side, the brightness is given by

$$
\begin{aligned}
I_{o}= & \frac{P_{I x} C}{4 \pi} \sigma_{x} \cos \theta_{o} \bullet \\
& \left\{\frac{1}{\sigma_{o} \cos \varphi-\sigma_{x} \cos \theta_{o}}\left[\exp \left(\tau \frac{\sigma_{o} \cos \varphi-\sigma_{x} \cos \theta_{o}}{\cos \theta_{o} \cos \varphi}\right)-1\right]+\right. \\
& \left.\frac{-R}{\sigma_{o} \cos \varphi+\sigma_{x} \cos \theta_{o}} \exp \left(\frac{2 \theta_{o} \tau}{\cos \theta_{o}}\right)\left[\exp \left(-\tau \frac{\sigma_{o} \cos \varphi+\sigma_{x} \cos \theta_{o}}{\cos \theta_{o} \cos \varphi}\right)-1\right]\right\}
\end{aligned}
$$

When $\theta_{0}=180 \mathrm{deg}, \phi=0 \mathrm{deg}$ and the reflectivity is zero,

$$
I_{o}=\frac{P_{x J} C}{4 \pi} \frac{\sigma_{x}}{\sigma_{o}+\sigma_{x}}\left\{1-\exp \left[-\tau\left(\sigma_{o}+\sigma_{x}\right)\right]\right\} .
$$

Equation 15 gives the intensity at a detector located on the back side of a front-side, $\mathrm{x}$-ray-exposed phosphor screen. Equation 15 would be applicable to a situation where the phosphor was coated on an optically transparent substrate with 
the phosphor toward the $\mathrm{x}$-ray beam and the substrate toward the detector. Equation 17 gives the intensity at a detector observing the $x$-ray illuminated side of the phosphor at normal or near-normal incidence.

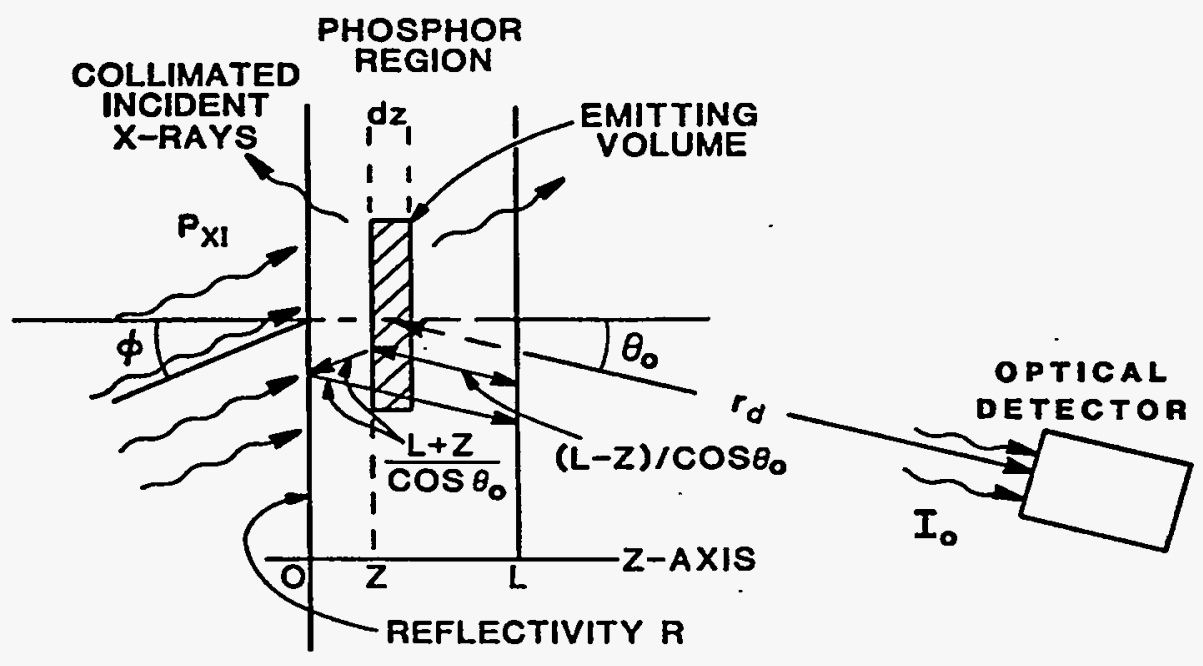

Figure 7. Geometry of x-ray-excited phosphor and detector.

If $s$ is the sensitivity of an imager (such as a camera) to an optical flux $F$ in, for example, $\mathrm{V} / \mathrm{W} / \mathrm{cm}^{2}$ (or $\mathrm{V} / \mathrm{J} / \mathrm{cm}^{2}$ ), the peak signal $S$ in volts of a system imaging the phosphor with a lens of $\mathrm{f}$-number $f$ is given by

$$
S=s F=s \frac{\pi}{4} \frac{1}{f^{2}} I_{0} .
$$

As an example, we can now estimate the peak camera signal for a system imaging the APS beam, where $E_{b}=7000 \mathrm{MeV}$ and $\rho=3900 \mathrm{~cm}$. It is assumed that the camera has an $f / 2$ lens viewing the back side of a WL-1201 screen which is imaging the beam at a magnification of 4 through a $10 \mu \mathrm{m}$ pinhole located $1400 \mathrm{~cm}$ from the beam. The spectrum is filtered to peak at $80 \mathrm{keV}$ through $0.5 \mathrm{~cm}$ of Be, $0.16 \mathrm{~cm}$ of $\mathrm{Al}$, and $0.035 \mathrm{~cm}$ of Mo. The filters reduce the total beam power by a factor of 22.6. During the initial operation, APS will run a $100 \mathrm{~mA}$ beam of 20 bunches at the ring frequency of $270 \mathrm{kHz}$. The initial beam will be $\sim 100 \mu \mathrm{m}$ in diameter. The $5.4 \times 10^{6}$ pulses per second will produce $910 \mathrm{~W} / \mathrm{cm}^{2}$ at the pinhole. After passing through the pinhole and filters, the incident $\mathrm{x}$-rays will produce an image at the phosphor with a peak x-ray flux $P_{X I}=2.5 \times 10^{-2} \mathrm{~W} / \mathrm{cm}^{2}$.

Figure 8 is a plot of $F / P_{X I} . F / P_{X I}$ is plotted both for a camera system imaging from the side opposite the $\mathrm{x}$-ray illumination ( $I_{o}$ given by Eq. 15$)$ and for a camera 
system imaging from the same side as the $\mathrm{x}$-ray illumination ( $I_{0}$ given by Eq. 17). The optimal thickness for the back-side case is $26 \mathrm{mg} / \mathrm{cm}^{2}$, where the value of $F / P_{X I}$ is $4.37 \times 10^{-7}$. The value of $F / P_{X I}$ for the front-side case asymptotically approaches a maximum value of $4.43 \times 10^{-7}$.

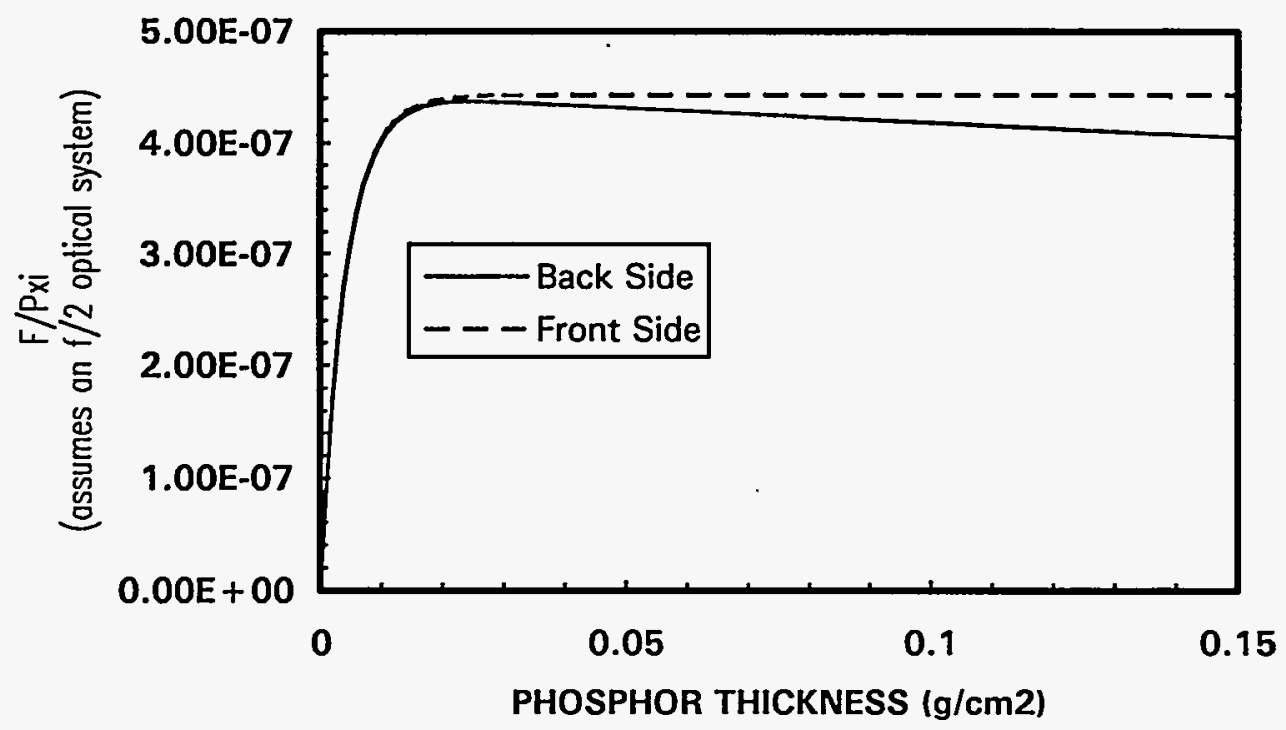

Figure 8. Optical flux at camera target normalized to $x$-ray flux at WL-1201 phosphor $\left(F / P_{X I}\right)$ for a camera observing from the back side or front side relative to the $\mathrm{x}$-ray illumination.

Assuming back-side observation, the optical flux $F$ at the camera target using the optimum thickness of phosphor is $1.1 \times 10^{-8} \mathrm{~W} / \mathrm{cm}^{2}$. Assuming a camera sensitivity of $10^{9} \mathrm{~V} / \mathrm{W} / \mathrm{cm}^{2}$ (similar to the camera used to image the optical synchrotron emission above), the signal would be $11 \mathrm{~V}$ if the camera did not saturate first.

The purpose of using a fast phosphor such as WL1201 is to image a single pulse out of several in a narrow macro pulse. In the case of some modes of operation at APS, there may be more than 20 bunches which would yield $>5.4 \times 10^{6} \mathrm{x}$-ray pulses per second. The bunches may not necessarily be evenly spaced about the ring, which would decrease the time between $x$-ray pulses and require nanosecond phosphor response. A single $18.5 \mathrm{nC}$ bunch produces $2.0 \times 10^{-15} \mathrm{~J} / \mathrm{cm}^{2}$ of optical flux at the camera for the conditions described above. This represents about 4000 photons $/ \mathrm{cm}^{2}$ at the camera target at the $400 \mathrm{~nm}$ emission wavelength of WL-1201. A camera with $40 \%$ quantum efficiency would record only 1600 events $/ \mathrm{cm}^{2} / \mathrm{bunch}$. An image formed with these few points would be granular and hard to interpret. In order to do single-pulse imaging it is necessary 
to use a phosphor more efficient than WL-1201 but still faster than the shortest bunch spacing. One could also increase the solid angle of the $x$-ray imager.

The phosphor information in this paper is by no means comprehensive and more efficient phosphors may exist. There are several prospects for increasing the solid angle of the x-ray imaging system; these however, have the disadvantage of added complexity over simple pinhole imaging. Larger pinholes may be used if the pinhole transfer function can be accurately measured. The beam can then be imaged and the measured point response of the pinhole can be used to numerically recover the beam profile. This technique requires a small source to characterize the pinhole. Henke-like sources exist that produce source sizes on the order of a few microns at $\sim 100 \mathrm{keV}$. The source is relatively weak and counting techniques must be used to do the characterization. Pinhole sizes greater than the beam profile can be used to do penumbral imaging, as is done with neutrons, thereby greatly increasing the solid angle(21). Other coded aperture techniques that have an increased solid angle are possible, including annular and zone plate imaging. These techniques require fabrication of a more complicated aperture, and the deconvolution methods often generate low-level image artifacts, which would complicate imaging of beam halos.

Equation 6 indicates that resolution of a system using optical elements is proportional to $\lambda^{2 / 3}$. Therefore a system imaging in $\mathrm{x}$-rays should produce improved resolution and still have large solid-angle acceptance. The difficulty of fabricating $\mathrm{X}$-ray optics often compromises the resolution a system obtains. The Kirkpatrick-Baez (K-B) microscope, which uses crossed grazing-incidence cylindrical mirrors, has successfully $\mathrm{x}$-ray imaged inertial-confinement laser targets with $5 \mu \mathrm{m}$ resolution at greater than $5 \mathrm{keV}$ x-ray energies(22). The K-B microscope has achieved acceptance angles of $\Delta \Omega=3 \times 10^{-7}$ ster as compared to the $4 \times 10^{-13}$ ster acceptance angle of the $10 \mu \mathrm{m}$ pinhole at $1400 \mathrm{~cm}$. Note that $(\Delta \Omega)^{1 / 2}=5.48 \times 10^{-4}>1 / \gamma=7.3 \times 10^{-5} \mathrm{rad}$, so that the K-B microscope acceptance is approximately greater than $\theta_{\mathrm{c}}$. The geometry of the $\mathrm{K}-\mathrm{B}$ microscope requires shorter optics-to-source distances for higher energy imaging where the required mirror angles are more glancing. The shorter distance increases difficulties from heat loading in the support structures, which may not be at a glancing angle. Imaging at lower energies would mitigate this problem.

Wölter $x$-ray microscope optics, consisting of consecutive hyperboloid and ellipsoid surfaces that are used to $\mathrm{x}$-ray image inertial-confinement laser targets, have been shown to give $\$ 10 \mu \mathrm{m}$ resolution, depending on the direction in the image plane relative to the optical axis(23). Unfortunately, Wölter optics are extremely difficult to fabricate.

\section{CONCLUSIONS}

Imaging of electron and positron beams using synchrotron emission is a useful diagnostic for determining beam position and profiles. Imaging in the visible range 
is a.useful technique for low energy machines down to beam energies of about 20 $\mathrm{MeV}$. Above this beam energy, there is ample light for single bunch imaging. The optical aperture and therefore optimal resolution are determined by balancing the blurring of the beam as it rounds a bend and diffraction of the optical aperture. For high-energy machines, because of the increased bending radius of the magnets, optical resolution in the visible range is $\gtrsim 150 \mu \mathrm{m}$. Resolution is proportional to $\lambda^{2 / 3}$ and therefore, imaging at shorter wavelengths is advantageous. Imaging at shorter wavelengths has the disadvantages of the difficulties associated with the fabrication of high-quality $\mathrm{x}$-ray components.

Simple pinhole imaging of high-energy beams is possible. Optimal resolution necessitates a compromise between diffraction through the pinhole and geometrical blurring from the finite pinhole diameter. The resolution is improved by imaging at higher energies. The problems are fabrication of $\sim 10 \mu \mathrm{m}$ pinholes thick enough to work at high energy, decreasing signal levels above the critical energy, low imaging efficiency due to the small solid angle of the pinhole, and the converter and imager technologies required. Imaging at TV rates is relatively easy. To image a single bunch with $\sim 30 \mu \mathrm{m}$ resolution is difficult because of the small signal levels. Various more sophisticated $\mathrm{x}$-ray imaging techniques discussed, such as coded imaging and $x$-ray optics, may increase signal levels but have the drawback of increased complexity.

\section{ACKNOWLEDGMENTS}

The author thanks David Bowman and Roddy Walton for engineering information and Peter Gobby for manufacturing information regarding the micro pinholes. I also thank S. Iverson and W. D. Turley for information regarding the WL-designation phosphors and Bingxin Yang and Alex Lumpkin for information regarding pinhole imaging at APS. Thanks to N. S. P. King for providing electrooptic imager information. Lastly, thanks to Vaikunth Stewart for the constructive editorial comment.

\section{REFERENCES}

1. Elder, F. R., Gurewitsch, A. M. and Langmuir, R. V., "Radiation from Electrons in a Synchrotron," Phys. Rev. 71, 829 (1947).

2. Nawrocky, R. J., Galayda, J., Yu, L. H. and Shu, D. M., "A Beam Profile Monitor for the NSLS VUV Ring Employing Linear Photodiode Arrays," IEEE Trans. Nucl. Sci. NS-32, 1893 (1985).

3. Hofmann, A., "Electron and Proton Beam Diagnostics with Synchrotron Radiation," IEEE Trans. Nucl. Sci. NS-28, 2132 (1.981). 
4. Greegor, R. B. and Lumpkin, A. H., 'Synchrotron Radiation as a Low Gamma, Non-Intercepting Diagnostic Concept for the Average Power Laser Experiment (APLE)," Nucl. Inst. Meth. A318, 422 (1992).

5. Krinsky, S., Bittner, J., Fauchet, A. M., Johnson, E. D., Keane, J., Murphy, J., Nawirocky, R. J., Rogers, J., Singh, O. V. and Yu, L. H., "Storage Ring Development at the National Synchrotron Light Source," BNL-46615, (Brookhaven National Laboratory, Sept. 1991), Ch. 2, pp. 14-20.

6. Hofmann, A. and Robinson, K. W., "Measurement of Cross Section of a High-Energy Electron Beam by Means of the X-ray Portion of the Synchrotron Radiation," IEEE Trans. Nucl. Sci. NS-18, 937 (1971).

7. Akbari, H., Borer, J., Bovet, C., Delmere, Ch., Manarin, A., Rossa, E., Sillanoli, M. and Spanggaard, J., "Measurement of Vertical Emittance at LEP from Hard X-Rays," in Proceedings of the 1993 Particle Accelerator Conference, (IEEE Wash. D.C. 1993), pp. 2492-2494.

8. Jackson, J. D.; Classical Electrodynamics (John Wiley \& Sons, Inc., New York, 1962), Ch. 14, pp. 481-488.

9. Feledman, D. W., Bender, S. C., Byrd, D. A., Carlsten, B. E., Early, J. W., Feldman, R. B., Goldstein, J. C., Martineau, R. L., O’Shea, P. G., Pitcher, E. J., Schmitt, M. J., Stein, W. E., Wilke, M. D. and Zaugg, T. J., "Operation of the APEX Photoinjector Accelerator at $40 \mathrm{MeV}$," in Proceedings of the 1992 Linear Accelerator Conference Proceedings, (AECL Research, Ottawa, 1992) pp. 603-605.

10. Armstrong, S. V. and Gobby, P. L., "Computer Aided Electroplating of Tapered Copper Electroforming Mandrels for Pinhole Imaging Components," Plating Surf. Finish., 76, 47 (1989).

11. Dunn, J., Young, B. K. F. and Shiromizu, S. J., "X-Ray Sensitive CCD Instrumentation for Short and Ultra-Short Pulse Laser-Produced Plasma Experiments," presented at the 10th Topical Conference on High-Temperature Plasma Diagnostics, May 8-12, 1994, Rochester, NY., Proc. to be published in Rèv Sci. Instrum., (1994).

12. Janesick, J,, Elliott, T. and Pool, F., "Radiation Damage in Scientific Charge-Coupled Devices," presented at the 1988 IEEE Nuclear Science Symposium, Orlando, Fl. Nov 11-19, (1988).

13. Ashford, C. B., Berlman, I. B., Flournoy, J. M., Franks, L. A., Iversen, S. G. and Lutz, S. S., "High-Z Liquid Scintillators Containing Tin," Nucl. Instr. Mëh., A243, 131(1986).

14. Moy, J. P., Koch, A. and Nielsen, M. B., "Conversion Efficiency and Time Response of Phosphors for Fast X-Ray Imaging with Synchrotron Radiation," Núcl. Instr. Meth., A326, 581 (1993).

15. Zurro, B., Ibarra, A., Acuña, A. U., Sastre, R: and McCarthy, K. J., "A Comparison of Phosphors for Broadband Plasma Emission Detectors," presented at the 10th Topical Conference on High-Temperature Plasma 
Diagnostics, May.8-12, 1994, Rochester, NY., Proc. to be published in Rev Sci. Instrum., (1994).

16. Shu, D., Warwick, T. and Johnson, E. D., "Diagnostic Phosphors for Photon Beams at the ALS and APS,", Rev. Sci. Instrum., 63, 548 (1992).

17. Electronic Industries Association (EIA) Tube Engineering Advisory Council (TEPAC), "Optical Characteristics of Cathode Ray Tube Screens," TEPAC Pub. No. 116, Electronic Industries Association, Wash., D.C. (Dec. 1980).

18. From the ITT Electro-Optical Products Brochure: "Special Purpose Photosensitive Devices," ITT Corp., (Jan. 1989).

19. Wilke, M., Iverson, S., Hockaday, M. and Blake, R., unpublished data.

20. Gleason, J. K. and Turley, W. D., "Photophysics and Chemistry of Ultra-Fast $\mathrm{ZnO}$ and CdS Phosphors,"Proc. 180th Meeting of the Electrochemical Society, Phoenix, AZ., Oct. 13-18, 1991.

21. Delage, O., Garconnet, J-P. and Schirmann, D., "Neutron Penumbral Imaging of Inertial Confinement Fusion Targets at Phébus," presented at the 10th Topical Conference on High-Temperature Plasma Diagnostics, May 8-12, 1994, Rochester, NY., Proc. to be published in Rev Sci. Instrum., (1994).

22. Marshall, F. J., Delettrez, J. A., Epstein, R. and Yaakobi, B., "Diagnosis of Laser-Target Implosions by Space-Resolved Continuum Absorption X-Ray Spectroscopy," Phys. Rev. E, 49, 4381 (1994).

23. Remington, B. A. and Morales, R. I., "Laboratory Characterization of Wölter X-Ray Optics," presented at the 10th Topical Conference on High-Temperature Plasma Diagnostics, May 8-12, 1994, Rochester, NY., Proc. to be published in Rev Sci. Instrum., (1994). 